\title{
Breaking the state of the heart: meshless model for cardiac mechanics
}

\author{
Èric Lluch - Mathieu De Craene - Bart Bijnens - Maxime Sermesant • \\ Jérôme Noailly • Oscar Camara • Hernán G. Morales
}

Received: date / Accepted: date

\begin{abstract}
Cardiac modeling has recently emerged as a promising tool to study pathophysiology mechanisms and to predict treatment outcomes for personalized clinical decision support. Nevertheless, achieving convergence under large deformation and defining a robust meshing for realistic heart geometries remain challenging, especially when maintaining the computational cost reasonable. Smoothed Particle Hydrodynamics (SPH) appears to be a promising alternative to the Finite Element Method (FEM) since it removes the burden of mesh generation. A point cloud is used where each point (particle) contains all the physical properties that are updated throughout the simulation. SPH was evaluated for solid mechanics applications in the last decade but its capacity to address the challenge of
\end{abstract}

\section{E. Lluch}

BCN MedTech, Universitat Pompeu Fabra, Barcelona, Spain Medisys, Philips Research, France

E-mail: eric.lluch@upf.edu

M. De Craene

Medisys, Philips Research, France

B. Bijnens

BCN MedTech, Universitat Pompeu Fabra, Barcelona, Spain

M. Sermesant

INRIA, Université Côte d'Azur, Sophia Antipolis, France

J. Noailly

BCN MedTech, Universitat Pompeu Fabra, Barcelona, Spain

O. Camara

BCN MedTech, Universitat Pompeu Fabra, Barcelona, Spain

H. G. Morales

Medisys, Philips Research, France simulating the mechanics of the heart has never been evaluated.

In this paper, a total Lagrangian formulation of a corrected SPH was used to solve three solid mechanics problems designed to test important features that a cardiac mechanics solver should have. SPH results, in terms of ventricle displacements and strains, were compared to results obtained with 11 different FEM-based solvers, by using synthetic cardiac data from a benchmark study. In particular, passive dilation and active contraction were simulated in an ellipsoidal left ventricle with the exponential anisotropic constitutive law of Guccione following the direction of fibers.

The proposed meshless method is able to reproduce the results of three benchmark problems for cardiac mechanics. Hyperelastic material with fiber orientation and high Poisson ratio allows wall thickening/thinning when large deformation is present.

Keywords Smoothed Particle Hydrodynamics $(\mathrm{SPH}) \cdot$ Finite Element Method (FEM) · meshless · cardiac mechanics - benchmark - hyperelastic exponential

\section{Introduction}

Cardiac modeling has recently emerged as a promising tool to study cardiac pathophysiology, integrate different echo measurements, for data augmentation and to predict treatment outcomes for personalized clinical decision support $[10,5]$.

The finite element method (FEM) appears as the golden standard numerical technique to solve the partial differential equations that simulate the cardiac multi-physics behavior [8]. Some of the reasons are its strong mathematical foundation, maturity and the 
availability of several academic and commercial software.

Cardiac tissue undergoes large deformations during the cardiac cycle due to physiological loads of myocardial active contraction and relaxation [23]. A cardiac in-silico model needs to be able to simulate characteristics observed in the heart's behavior such as these large deformations. Some finite element simulations in cardiac tissue mechanics were able to mimic the heart behavior in a realistic way [8, 38]. Simulations have even been personalized to assimilate heart displacements from medical images and generate synthetic images $[31,12]$. However, several challenges arise when using FEM. The most critical one is the inherent need of a mesh and efficient remeshing strategies.

To overcome mesh-related issues, recent articles have tackled the fluid-structure interaction (FSI) in the context of cardiac modeling by combining two numerical techniques, such as FEM for solid mechanics and smoothed particle hydrodynamics (SPH) for fluids [30]. However, these approaches require to excel in both numerical techniques, as well as the specific implementation for the fluid solid interaction. A simpler approach will be to have different multi-physics phenomena modeled with the same numerical scheme. For FSI simulations, SPH has been used in the past for simple problems. SPH simulations in the context of FSI were able to properly reflect laboratory measurements [2] and to reproduce numerical benchmarks [17]. Moreover, SPH has already been used for cardiac electrophysiology [27]. SPH was chosen over other meshless methods for its versatility that would allow for a multi-physics cardiac model. Among other advantages, SPH is easy to parallelize, it is memory efficient and its improved version can account for large deformations while limiting convergence problems [41].

$\mathrm{SPH}$ is a meshless Lagrangian method to solve partial differential equations numerically. Instead of requiring a mesh-based discretization of the spatial domain, a point cloud describing the geometry with the material physical properties defined at each point is sufficient.

Original formulation of meshless methods is known to suffer from convergence issues [3]. In the case of SPH, the first application in solid mechanics dates back to 1991 [25]. Nevertheless, further use of SPH for solid mechanics were originally limited by three drawbacks. First, SPH particles tended to cluster locally making appear non-physical gaps between particles [13, 39, 32]. Second, the original formulation of SPH could lead to non-physical solutions due to numerical instability produced by its rank-deficiency $[4,36]$. And third, SPH original formulation did not provide first order completeness implying numerical errors in the computa- tion of gradients of constant and linear fields [9]. In the last decade, these three challenges have been overcome through the use of a total Lagrangian SPH [7] and normalization of the gradients [9]. Therefore, only recently SPH has been successfully applied in the context of solid mechanics. As an example, SPH was successfully compared to FEM for tissue rupture simulations [37], and could simulate large deformations when a pure Lagrangian formulation was used [34].

The purpose of this paper is to demonstrate that SPH can be used to efficiently tackle the challenges specific to the modeling of cardiac tissue mechanics: complex geometry given by an arbitrary point cloud, fibresinduced anisotropy, tissue incompressibility, nonlinear elasticity and large deformations. This is the first application of SPH to perform analysis of cardiac mechanics using a nonlinear constitutive model and a simple contraction model. But it is not the first time that a simulation of cardiac mechanics using a meshfree method is used [40].

Specifically, SPH was adapted to cope with large deformations in a three dimensional ellipsoidal body in the context of cardiac mechanics, which was not available until now in the literature. Applying the SPH framework in this very specific context led us to introduce three main algorithmic features.

- First, a total Lagrangian formulation that translates Guccione's hyperelastic material into SPH was derived [16]. This is key to handle the large deformations undergone by the cardiac tissue.

- Second, a generalization of SPH to an arbitrary geometry was presented. Given any set of points in the space within a known continuum body, cell volumes and surface areas for each point need to be determined before starting any SPH simulation.

- Third, a damping Rayleigh-type control was added to damp possible extra vibrations generated from the inertial acceleration when dynamic simulations were performed in continuum mechanics [34].

\section{Total Lagrangian formulation in solid mechanics}

Let us consider the $3 D$ deformation of a continuum isothermal body ${ }^{1}$ that moves from a reference configuration, defined by the positions $\mathbf{X} \in \Omega_{0} \subset \mathbb{R}^{3}$ and the boundary surface $\partial \Omega_{0} \subset \mathbb{R}^{2}$, to a current configuration at time $t$ defined by the positions $\mathbf{x} \in \Omega_{t} \subset \mathbb{R}^{3}$ and surface boundary $\partial \Omega_{t} \subset \mathbb{R}^{2}$. In the total Lagrangian

1 When the difference of temperature is taken into account a thermal energy equation should be added in Eq. (2) 
formulation, the reference configuration remains unchanged as the simulation proceeds and it is only used to compute stresses and accelerations. Current positions and velocities are updated and points move according to the accelerations computed based on the reference configuration.

The body motion at time $t$ is described as a mapping $\phi: \Omega_{0} \mapsto \Omega_{t}$ between the reference coordinates, and the current coordinates:

$\mathbf{x}=\phi(\mathbf{X}, t)$

The displacement between configurations is given by $\mathbf{U}=\mathbf{x}-\mathbf{X}$. Since the body is considered to be isothermal, and dissipative effects are neglected, the dynamics of the motion $\phi$ satisfy the following set of conservation laws:

$$
\begin{aligned}
\rho J & =\rho_{0}, \\
\frac{d^{2} \mathbf{U}}{d t^{2}} & =\frac{1}{\rho_{0}} \operatorname{div}(\mathbf{P})+\mathbf{B}=\frac{1}{\rho_{0}} \nabla_{0} \cdot(\mathbf{P})+\mathbf{B},
\end{aligned}
$$

where $\cdot$ is the dot product, $\rho$ and $\rho_{0}$ are current and reference densities, respectively, $J$ is the determinant of the deformation gradient $\mathbf{F}$

$J=\operatorname{det}(\mathbf{F}), \quad \mathbf{F}=\nabla_{0} \mathbf{x}=\frac{\partial \mathbf{x}}{\partial \mathbf{X}}=\frac{\partial \mathbf{U}}{\partial \mathbf{X}}+\mathbf{I}$

with $\mathbf{I} \in \mathbb{R}^{3,3}$ the identity matrix. $\mathbf{P}$ is the first PiolaKirchhoff stress tensor, $\mathbf{B}$ is the tensor corresponding to any extra body forces acting as boundary conditions and $\nabla_{0}$ is the material gradient operator, defined as $\nabla_{0} \equiv \frac{\partial}{\partial \mathbf{X}}$.

\subsection{Cardiac mechanics formulation}

To define $\mathbf{P}$, the constitutive equation needs to consider a strain energy density function $\psi$. This equation aims to describe the relation between strain and stress for the given material. Experiments in cardiac tissue suggest that the best mathematical model to characterize the constitutive material of the heart is with either hyperelastic or viscoelastic material [8]. In this paper, the transversely isotropic constitutive Guccione's law was used as a constitutive equation [16]. This hyperelastic law is solely exponential and linear terms are not present as it can be seen in Equation (4)

$\psi=\frac{C}{2}\left(e^{Q}-1\right)$

with
$Q=\left(\mathbf{b} \circ \mathbf{E}_{f}\right): \mathbf{E}_{f}$,

where $\circ$ and : denote the Hadamard and Frobenius inner product, respectively, $\mathbf{b}$ and $\mathbf{E}_{f}$ are defined as

$\mathbf{b}=\left(\begin{array}{ccc}b_{f} & b_{f s} & b_{f s} \\ b_{f s} & b_{t} & b_{t} \\ b_{f s} & b_{t} & b_{t}\end{array}\right)$

$\mathbf{E}_{f}=\mathbf{R}^{T} \mathbf{E R}=\mathbf{R}^{T} \frac{1}{2}\left(\mathbf{F}^{T} \mathbf{F}-\mathbf{I}\right) \mathbf{R}$,

where

$\mathbf{E}=\frac{1}{2}\left(\mathbf{F}^{T} \mathbf{F}-\mathbf{I}\right)$

is the Green-Lagrange strain tensor. $\mathbf{E}_{f}$ is $\mathbf{E}$ expressed in a local orthonormal coordinate system with fibres in the $f$-direction, $\mathbf{R}$ is the rotation tensor in the reference configuration given by $\mathbf{R}=\left[\mathbf{f}_{0}, \mathbf{t}_{0}, \mathbf{l}_{0}\right]$, where $\mathbf{f}_{0}$ is a normalized column vector with the fibre direction information, $\mathbf{t}_{0}$ is the transmural direction and $\mathbf{l}_{0}$ is the long-axis direction. Material properties are characterized by the parameters $C, b_{f}, b_{t}, b_{f s}$ and will be defined for each problem.

By deriving equation (4) with respect to $\mathbf{E}_{f}$ we obtain the first Piola-Kirchhoff tensor $\mathbf{P}$ as

$\mathbf{P}=\mathbf{F} \frac{\partial \psi}{\partial \mathbf{E}_{\mathbf{f}}}=\mathbf{F R}\left(\mathbf{b} \circ \mathbf{E}_{f}\right) \mathbf{R}^{T} C e^{Q}$.

A pressure-type Lagrange multiplier $p$ was introduced in the constitutive equation to handle incompressibility such that the first Piola-Kirchhoff stress tensor was written as

$\mathbf{P}=\mathbf{F} \frac{\partial \psi}{\partial \mathbf{E}_{\mathbf{f}}}-p \mathbf{F}^{-T}$

where

$p=K(J-1)$

with $K$ the bulk modulus that controls the elasticity of the material and is related to the Poisson ratio $\nu$ by the following equation:

$K=\frac{2 \mu(1+\nu)}{(3(1-2 \nu))}$

where $\mu$ is the shear modulus of the material.The closer to 0.5 the value of $\nu$ is, the stronger the incompressibility condition will be. A Poisson ratio of 0.495 was used 
by default for all simulations since it was enough to handle near incompressibility of the cardiac tissue experimentally.

Finally, for the complete definition of the Initial Boundary Value Problem (IBVP), initial and boundary (essential and natural) conditions must also be specified accordingly. These conditions will be further explained in Section 4 for each experiment but in general, a traction boundary condition will be applied on surfaces.

\section{$3 \mathrm{SPH}$ formulation}

In this section, the SPH method is introduced. Then, the above total Lagrangian formulation (Equation 2) is discretized using a corrected version of SPH.

From here onwards, subscript indices $i$ and $j$ denote two different particles, which are collocation points where the numerical solution of the equations of interest is computed. SPH physical properties are interpolated over a particle's immediate neighborhood. Given a real function $f(\mathbf{X})$ at $\mathbf{X}_{i}$, its value can be expressed as the convolution

$f\left(\mathbf{X}_{i}\right)=\int_{\Omega_{0}} f(\mathbf{X}) \delta\left(\mathbf{X}-\mathbf{X}_{i}\right) d \mathbf{X}$

where $\delta$ is the Dirac delta function. The first SPH approximation is to consider a kernel function $W \approx \delta$ in Equation (13). Kernel functions $W$ have usually finite support, controlled by the smoothing length $h$ in order to better approximate the Dirac delta function, which has zero support. Therefore, $W$ must be chosen to satisfy the unity and compact support properties and should converge to the Dirac delta function for smoothing lengths $h \rightarrow 0$. Depending on the equation to be discretized, the kernel can be defined so that its first or second derivative are positive everywhere.

The second approximation in the SPH framework is to estimate the integral expression in Equation (13) with a finite sum over discrete evaluations of the function $f_{j}=f\left(\mathbf{X}_{j}\right)$ of each particle that lies in the neighborhood $N_{i}$ of $i$. These two approximations lead to the following mathematical definition of a function $f$ for a given particle $i$ and neighbors $j$ in its neighborhood $N_{i}$.

$f_{i}=\sum_{j \in N_{i}} f_{j} V_{j} W_{i}\left(\mathbf{X}_{i}-\mathbf{X}_{j}, h\right)$

where $W_{i}\left(\mathbf{X}_{i}-\mathbf{X}_{j}, h\right)$ is a kernel function depending only on the scalar distance between particles $i$ and $j, V$ denotes the particle volume in the initial configuration.
An advantage of the SPH formulation is that the referential gradient of $f(\mathbf{X})$ at particle $i$ does not depend on the derivative of $f_{j}$ :

$\nabla_{\mathbf{X}} f\left(\mathbf{X}_{i}\right)=\sum_{j \in N_{i}} f_{j} V_{j} \nabla_{\mathbf{X}} W_{i}\left(\mathbf{X}_{i}-\mathbf{X}_{j}, h\right)$,

The disadvantage of this formulation is that gradients of constant or linear functions are not correct, and they do not ensure conservation of linear and angular momentum, respectively $[7,6]$. To remove this deficiency, a Corrected Smoothed Particle Method (CSPM)-correction has been proposed [9]. This is intuitively a correction of the integration domain that accounts for the number of neighbours inside the kernel size thanks to a first order Taylor approximation. At the beginning of the simulation, the first-order correction to derive the correcting tensor $\mathbf{K}$ for each particle $i$ is computed:

$\mathbf{K}_{\mathbf{i}}=\sum_{j \in N_{i}} V_{j} \nabla W_{i}\left(\mathbf{X}_{i}-\mathbf{X}_{j}, h\right) \otimes\left(\mathbf{X}_{j}-\mathbf{X}_{i}\right)$,

where $\otimes$ represents the dyadic product of two vectors. $\mathbf{K}$ is used to correct the gradient operator with respect to the initial configuration: [9]

$\tilde{\nabla}_{0} W_{i}\left(\mathbf{X}_{i}-\mathbf{X}_{j}, h\right)=\mathbf{K}_{i}^{-1} \nabla_{0} W_{i}\left(\mathbf{X}_{i}-\mathbf{X}_{j}, h\right)$

\subsection{Total Lagrangian SPH in solid mechanics}

Having the SPH approximations for a function $f$ and its gradient evaluated in a given particle $i$, equations (2) can be expressed as follows:

$$
\rho_{i}=\frac{\rho_{0}}{J_{i}},
$$

$$
\begin{aligned}
& \frac{\partial^{2} \mathbf{U}_{i}}{\partial^{2} t}=\frac{1}{\rho_{0}} \sum_{j \in N_{i}} V_{j}\left(\mathbf{P}_{j}-\mathbf{P}_{i}-\mathbf{\Pi}_{\mathbf{0} i j}\right) \\
& \tilde{\nabla}_{0} W_{i}\left(\mathbf{X}_{i}-\mathbf{X}_{j}, h\right)+\mathbf{B}_{i}, \\
& \mathbf{F}_{i}=\sum_{j \in N_{i}} V_{j}\left(\mathbf{x}_{j}-\mathbf{x}_{i}\right) \tilde{\nabla}_{0} W_{i}\left(\mathbf{X}_{i}-\mathbf{X}_{j}, h\right),
\end{aligned}
$$

where an artificial viscosity term $\boldsymbol{\Pi}_{\mathbf{0}}$ ij was added in Eq (18b), which is necessary to simulate large deformations. The artificial viscosity term is typically used in the SPH to stabilize numerical artefacts originated from the SPH formulation such as the reduction of unphysical oscillations [33]. The artificial viscosity in the initial configuration is given by $\boldsymbol{\Pi}_{\mathbf{0} i j}=J \Pi_{i j} \mathbf{F}^{-T}$ with 
$\Pi_{i j}= \begin{cases}\frac{-\alpha \bar{c}_{i j} \phi_{i j}+\beta \phi_{i j}^{2}}{\bar{\rho}_{i j}}, & \phi_{i j}<0 \\ 0, & \phi_{i j} \geq 0\end{cases}$

with

$$
\begin{aligned}
\phi_{i j}=\frac{\bar{h}_{i j}\left(\mathbf{v}_{i}-\mathbf{v}_{j}\right) \cdot\left(\mathbf{x}_{i}-\mathbf{x}_{j}\right)}{\left|\mathbf{x}_{i}-\mathbf{x}_{j}\right|^{2}+0.01 \bar{h}_{i j}^{2}} ; & \bar{\rho}_{i j}=\frac{\rho_{i}+\rho_{j}}{2} ; \\
\bar{c}_{i j}=\frac{c_{i}+c_{j}}{2} ; & \bar{h}_{i j}=\frac{h_{i}+h_{j}}{2}
\end{aligned}
$$

where $v$ is the particle velocity, $c$ is the so called wave speed related to the stiffness of the material. Since the total Lagrangian formulation overcomes numerical instabilities, the coefficients of artificial viscosity are chosen to be small to reduce its artificial effect. In particular $\alpha=0.04$ and $\beta=0.08$ were chosen following [26].

The $C^{2}$-Wendland Kernel will be used for the SPH formulation.

$W\left(\mathbf{X}_{i j}, h\right)=\frac{21}{2 \pi} \begin{cases}\left(1-\left|\mathbf{X}_{i j}\right|\right)^{4}\left(1+4\left|\mathbf{X}_{i j}\right|\right), & \left|\mathbf{X}_{i j}\right|<h \\ 0, & \text { otherwise }\end{cases}$

where $\mathbf{X}_{i j}$ is a variable for evaluation which typically takes the value $\left(\mathbf{X}_{i}-\mathbf{X}_{j}\right)$.

\subsection{Temporal discretization}

In this study, Runge Kutta (RK4) was used as a temporal discretization scheme. It has been shown that RK4 provides a good trade-off between speed and accuracy compared to other time discretization schemes since the Courant-Friedrichs-Levy (CFL) condition makes the time step choice less strict [28]. RK4 provides higher accuracy than the Euler scheme, which is the time discretization approach employed in the original formulation of SPH.

Due to the dynamic nature of equations (2), inertial acceleration is taken into account and undesirable vibration might appear. Therefore, a Rayleigh damping model was used to achieve the quasi-static solution [34]. In the case of SPH, this model imposes a condition on the time integration step

$\Delta t=\gamma \min \left(\frac{h}{c}\right)$

where $c$ is the speed of sound, $h$ is the smoothing length and $\gamma$ is a scale factor that is typically set to a value of 0.9 [34].
3.3 Boundary conditions and fiber definition

The application of boundary conditions within the SPH framework is typically limited to imposed velocity or displacement using fictitious ghost particles to compensate for the lack of particles beyond the boundary interface $[2,33]$. For solid mechanics there is a clear need to impose stresses upon boundaries. In order to impose these conditions in the total Lagrangian framework, the Nanson's relation between the reference and current areas is necessary

$d a \mathbf{n}=J d A \mathbf{F}^{-T} \cdot \mathbf{N}$

where $d a$ and $d A$ are the differential of areas in the current and reference configuration, respectively [35]. $\mathbf{n}$ and $\mathbf{N}$ are the surface normal vectors in the current and reference configurations, respectively. To define the direction of the applied loads, a binary function was defined for each particle, equal to 1 for the particles of the surface where the loads apply and equal to 0 for the rest of the particles. The gradient of this function was then calculated using the SPH formulation. In this way, the normal to the surface was computed [11], and the fiber orientation was defined as described in the paper $[22]$.

\subsection{Remarks on dynamic model}

Static solutions are required for this study, since comparisons with FEM are performed in the experiment section. Two adaptations of our dynamic model were made for quasi-static problems. First, traction boundary conditions were gradually increased until they reached the desirable maximum value. This strategy is commonly used to reduce the influence of inertia on the results. In particular, the pressure at each time step $p_{t}$ was increased gradually during $t_{1}=1 \mathrm{~s}$ of simulation until $p_{f}$ was reached. Then $p_{t}$ was set to $p_{f}$ and the simulation ran until $t_{2}=1.2 \mathrm{~s}$ to let enough time for the simulation to stabilize.

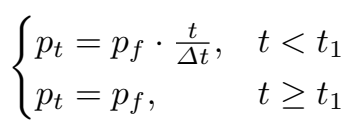

where $t$ is the current time of the simulation and $\Delta t$ is the time step.

Second, incompressible materials cannot be used in dynamic problems, because the speed of elastic waves becomes infinite [1]. Thus, nearly incompressible materials were used in this paper (see Equation (10)). 


\section{Experiments}

The experiments corresponded to the benchmark problems proposed by Land et al. [22]. In that benchmark, 11 FEM solvers were compared and results are available at [21].

The benchmark problems consisted in a defected bar, an idealized inflated ventricle and an idealized inflated ventricle with active stresses and oriented fibres. All these problems used the Guccione constitutive equation and were statically solved. Evaluations were purely numerical since no analytical solution exists.

Qualitative and quantitative evaluations were performed in the same way as presented in the benchmark [22]. Qualitatively, four stages during deformation are presented, until the maximum value of the stress boundary condition is reached. Quantitatively, three morphological descriptors were provided: centerline displacement, strains along the centerline and position of points of interest in space. Details of strain definition and these points are provided for each problem.

The simulations of the solver glasgowHeart-IBFE [15] with the highest number of degrees of freedom were taken here as reference for qualitative and centerline comparisons. This model was chosen since it used a high quality hexahedral mesh. The points of the glasgowHeart-IBFE mesh were used for all SPH simulations in this section except for the case of the bar, where a convergence analysis was performed on different number of particles (see Table 1). For the rest of the experiments the SPH solution was compared to the solutions provided by the 11 solvers of the benchmark.

For each problem, the strain $s_{i}$ was calculated tracking changes in the distance between pairs of $n$ points with coordinates $X_{1}^{i}$ and $X_{2}^{i}$ in the reference configuration and $x_{1}^{i}, x_{2}^{i}$ in the deformed configuration, where $i=0,1, \ldots, n$.

$s_{i}=\left(\frac{\left\|x_{1}^{i}-x_{2}^{i}\right\|}{\left\|X_{1}^{i}-X_{2}^{i}\right\|}-1\right) \times 100 \%$.

For all experiments, the time step was $1 e^{-5}$.

\subsection{Problem 1: Deformation of a beam}

First, a deflected rectangular beam in 3D was evaluated. Pressure-type follower forces whose directions and magnitude change with the deformed surface orientation were tested, as well as the correct implementation of fibre directions. The beam has a length of $10 \mathrm{~mm}$ and a rectangular cross-section of $1 \mathrm{~mm}^{2}$. A pressure of $4 P a$ was applied to the entire bottom face $(z=0)$. Fibers were oriented along the beam longitudinal axis, i.e. $(1,0,0)$. The constitutive parameters were $C=2 k P a, b_{f}=8, b_{t}=2, b_{f s}=4[22]$.
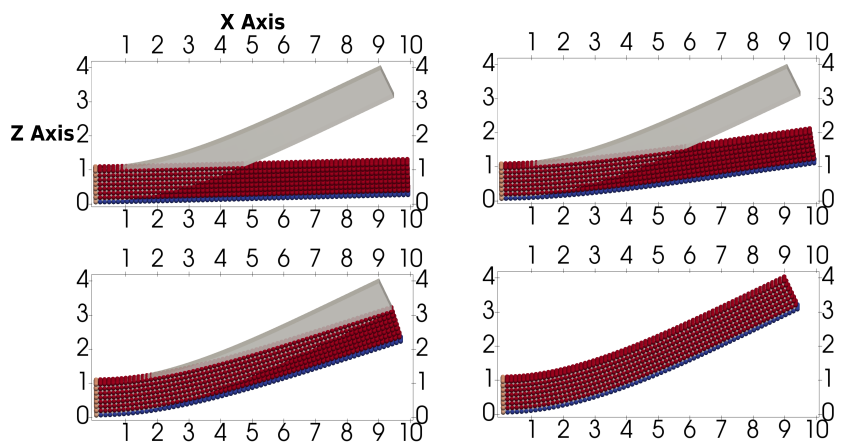

Fig. 1: Dynamic simulation of a hyperelastic bar using SPH with pressure imposed on surface given by blue particles. The selected deformed mesh computed using FEM (in grey) is plotted as a reference solution. Pressure is increased gradually from phase A to phase D until $4 P a$ where the final state is reached. A resolution of 4544 particles was used for the SPH simulation.

As qualitative results, four stages of deformation of the beam while increasing pressure are presented in Fig. 1. There, the blue particles are affected by the external pressure. A layer of particles on the left was fixed to model the clamped edge of the beam. The steady state was reached when the maximum pressure value was imposed and coincides with the deformed solution of the mesh-based solvers used in the benchmark.

The above observations were confirmed when quantifying deformations on the beam's centerline (Fig. 2)
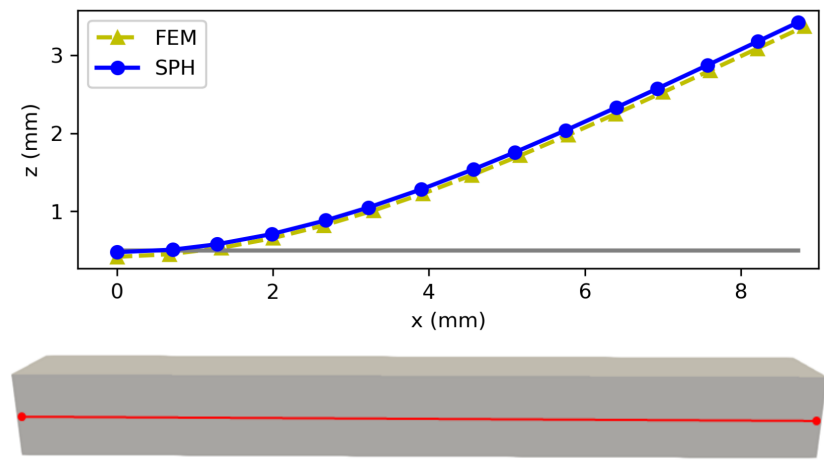

Fig. 2: Problem 1. Up: Comparison of the centerline in the mid of the bar before deformation (in grey) and after deformation for both FEM and SPH methods. A resolution of 4544 particles was used for the SPH simulation. Bottom: Undeformed mesh (in grey) with centerline (in red). 
and highest $z$ position (Fig. 3) with respect to the number of degrees of freedom. In Fig. 3, the $\mathrm{z}$ position of all FEM solution is provided. The $\mathrm{z}$ highest point computed with SPH lies within the range of values obtained with the finite element simulations. At the same time, a mesh convergence study was performed by maintaining to 3 the minimum number of neighbors associated to the SPH particles while increasing the number of particles in the point cloud (see Table 1). The value of the maximum z-deflection $4.16 \mathrm{~mm}$ was used for the computation of the relative difference in Table 1 since it is the value to which most of the FEM solutions with highest degrees of freedom converge (see Fig. 3).

\begin{tabular}{|l|l|l|l|l|l|}
\hline $\mathrm{n}^{\mathrm{o}}$ Particles & 1025 & 1836 & 4544 & 9100 & 15984 \\
\hline Z-deflect. $(\mathrm{mm})$ & 4.707 & 4.404 & 4.217 & 4.153 & 4.152 \\
\hline Rel. diff. (\%) & 13.15 & 5.87 & 1.37 & 0.17 & 0.19 \\
\hline
\end{tabular}

Table 1: Convergence analysis on the highest $\mathrm{z}$ point of the deformed beam for five different number of particles in the SPH point cloud. The third row shows the relative difference of the highest $\mathrm{z}$-deflection with respect to the value $4.16 \mathrm{~mm}$.

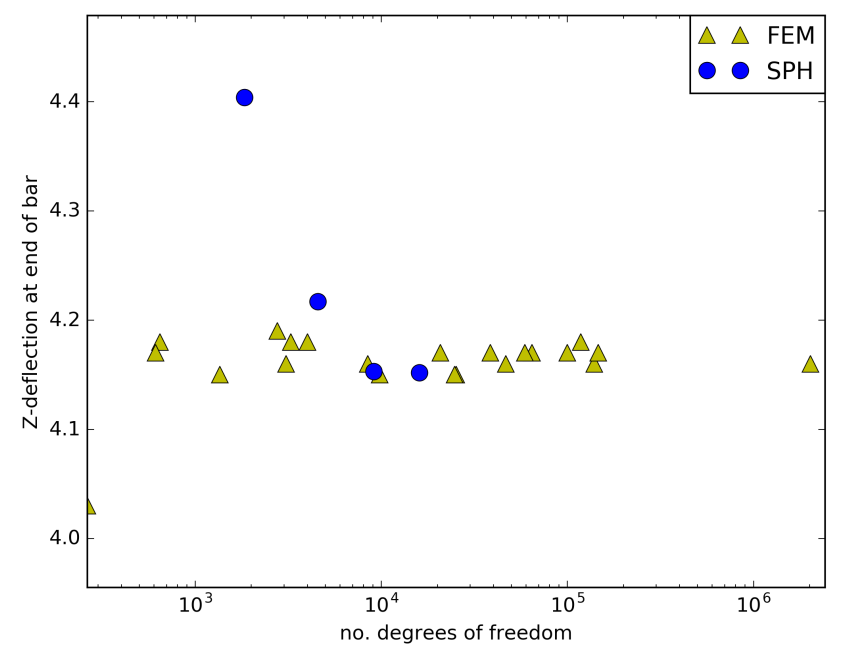

Fig. 3: Highest $\mathrm{z}$ point of the deformed beam for various FEM and SPH solutions depending on the degrees of freedom.

The strain was computed along the object's centerline in all three directions $x, y$ and $z$ (see Fig. 4). The index of points indicated on the horizontal axis corresponded to the $x$ coordinates in the undeformed configuration from left to right, every $1 \mathrm{~mm}$, as defined in the benchmark [22]. The strain curves are similar for both
FEM and SPH methods with differences of up to $0.01 \%$ in the $\mathrm{x}$-axis direction, $0.05 \%$ in the $\mathrm{y}$-axis direction and up to $0.32 \%$ in the z-axis direction.

\subsection{Problem 2: Inflation of a ventricle}

Second, an inflation of an ellipsoidal ventricle with isotropic material properties was evaluated [22]. This problem tests the discretization of a geometry described by an analytical expression and a deformation mimicking cardiac relaxation. The ventricle is given by an ellipsoid with a wall width of $10 \mathrm{~mm}$ and a length of $25 \mathrm{~mm}$. The analytical expression of the geometry can be found in [22]. A pressure of $10 k P a$ was applied on the endocardial surface and the basal plane $(z=5 \mathrm{~mm})$ was fixed in all directions. Finally, the constitutive parameters were set to $C=10 k P a, b_{f}=b_{t}=b_{f s}=1$ following instructions from the benchmark [22].

As qualitative results, four stages of the ventricle inflation while increasing pressure are presented in Fig. 5. The blue particles on the endocardium are affected by the pressure, which works as the blood pressure acting on a ventricle. The steady state was reached when the maximum pressure value was equal to the imposed value.

As it can be seen in Fig. 5 the SPH solution converged to the FEM solution. The model was able to simulate quasi-incompressibility keeping the same volume of the tissue, implying therefore, a wall thinning. This is especially noticeable by comparing Fig. $5 \mathrm{~A}$ with Fig. 5D where the thickening of the wall around the apex is reduced by about $50 \%$.

These qualitative results of the final deformation are confirmed by the long-axis centerline computation in Fig. 6 as well as the highest and lowest $z$ values in the apical region in Fig. 7. Specifically for Fig. 7, the $z$ position of all FEM solutions for different degrees of freedom is provided. The endocardial and epicardial $z$ position lies within the range of values obtained with the finite element simulations.

For the ellipsoidal problems, longitudinal, circumferential and transmural strains were calculated at the endocardium, epicardium and midwall as done in the benchmark [22]. Points on the long-axis centerline were used to compute the strains. For an exact definition of the points we refer to the benchmark [22]. Fig. 8 shows that both solutions have similar strain curves in terms of both behavior and range of values. The strain curve that showed most similar results with both methods is the circumferential strain in the epicardium and the one that obtained higher percentage of differences is the longitudinal strain curve in the endocardium (with up to $5 \%$ of differences in several points). 

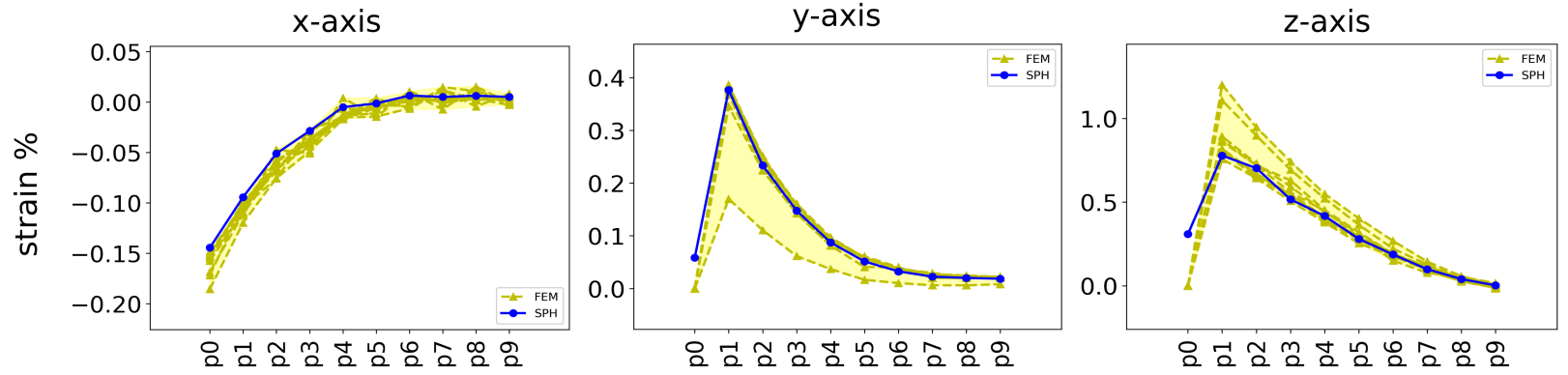

Fig. 4: Comparison of strain curves computed with the difference in positions $(p 0 \ldots p 9)$ between the initial and end configurations for both FEM and SPH solutions. Solutions from all 11 FE solvers published in [22] are overlaid with our SPH solution, where a resolution of 4544 particles was used.
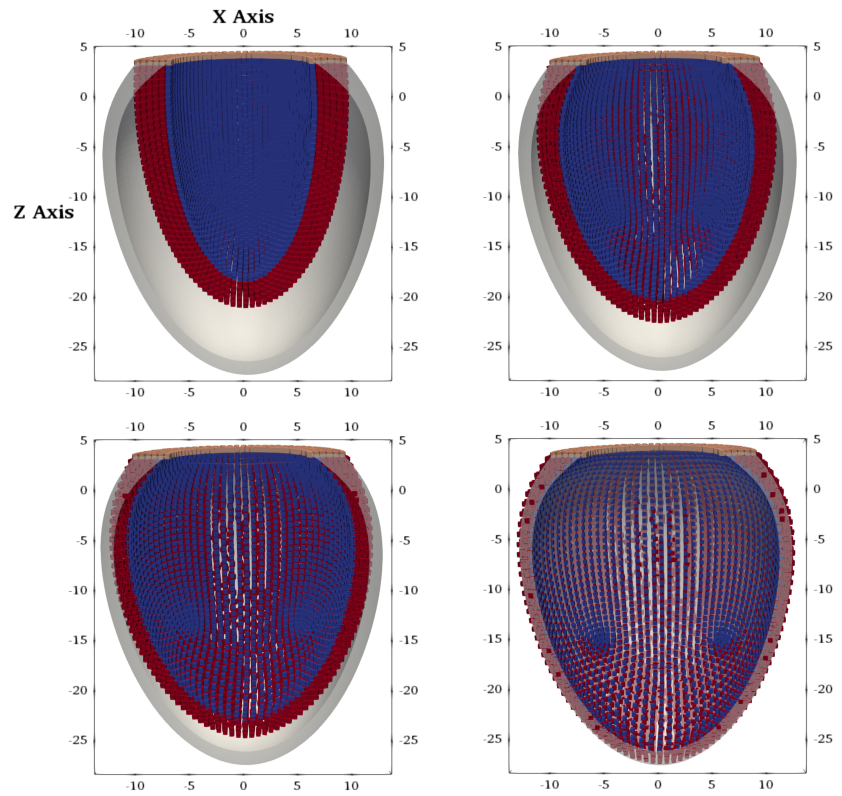

Fig. 5: Dynamic simulation of a ventricle inflation using $\mathrm{SPH}$ with the selected FEM solution as a reference (in grey). Endocardial pressure boundary conditions is applied on particles in blue. Pressure is increased gradually from phase A to phase D until $10 k P a$ where the final state is reached.

4.3 Problem 3: Inflation and active contraction of a ventricle

The third problem is the inflation and active contraction of an ellipsoid with transversely isotropic material properties. The problem tests reproducibility of complex fibre patents and the implementation of active contraction, both important aspects of a cardiac mechanics solver [22]. The reference geometry of this problem is exactly the same as in Problem 2. The pressure applied to the endocardium is of $15 k P a$, the basal plane

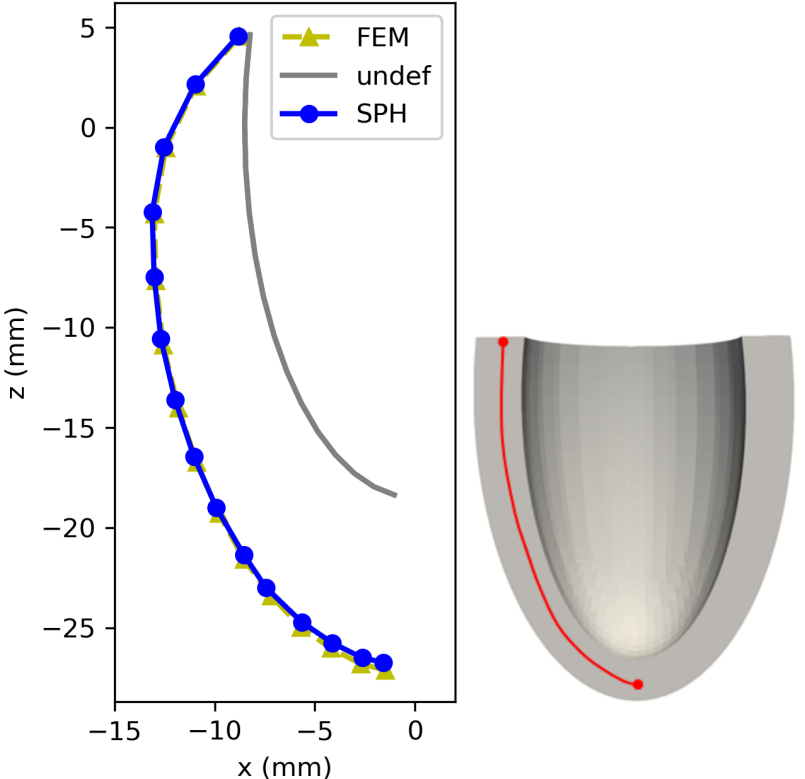

Fig. 6: Left: Comparison of the longitudinal centerline of problem 2 before (in grey) and after deformation for the SPH (in blue) and for the FEM (in dashed yellow line) simulations. Right: Undeformed mesh (in grey) with centerline (in red).

was also fixed and the constitutive parameters were the same as in Problem 1. Moreover, a system of fiber directions were defined on the myocardium and an active force was imposed on these fibres. The fibre angles $\alpha$ range from $-90^{\circ}$ at the epicardial surface to $+90^{\circ}$ at the endocardial surface. For a complete mathematical definition of the fibres we refer to [22].

Wall thickening is present at the end of the simulation of problem 3 due to the contractile forces and the incompressibility constraint (see Fig. 9 D). Rotation due to the fibre orientation is also visible when comparing the particle orientation of Fig. 9A against Fig. 9D. 


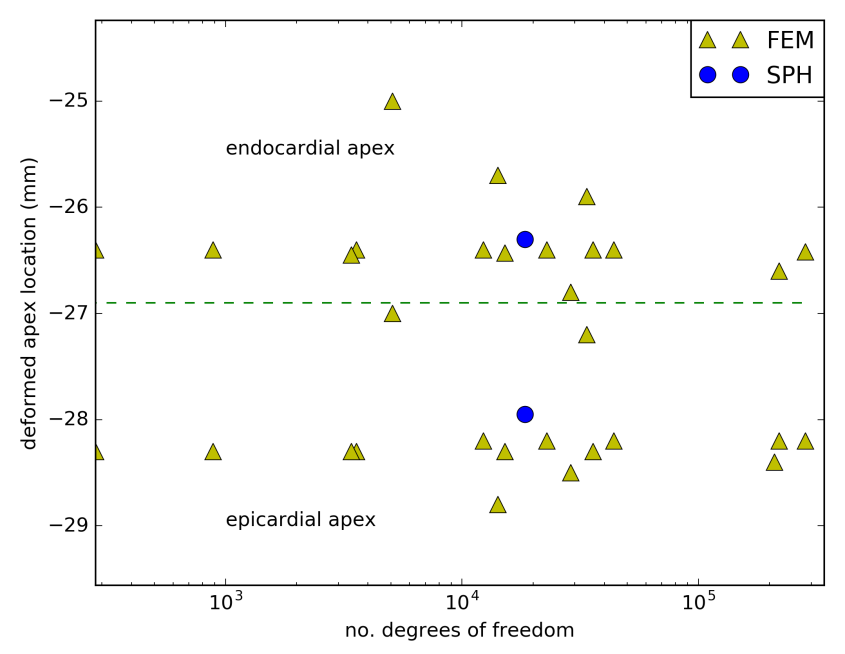

Fig. 7: Endocardial and Epicardial apex points for FEM and SPH simulations of problem 2 with different degrees of freedom.

These qualitative results of the final deformation are confirmed by the centerline computation in Fig. 10 as well as the highest and lowest $z$ values in the apical region in Fig. 11.

Specifically for Fig. 11, the $z$-position of all FEM solutions for different degrees of freedom is provided. The centerline has a stronger deformation for the SPH than for FEM.

The third problem is the one with higher differences between FEM and SPH strain curves, especially in strains of the midwall (see Fig. 12). But the SPH solution still lies among the median of the FEM solutions. The SPH solution achieves a slightly higher rotation than the one obtained using FEM (see Fig. 13).

Finally, three tables of quantitative differences between two compared models using the same geometry are shown in Tables 2,3 and 4.

\begin{tabular}{|c|c|c|c|}
\hline & $\mathrm{x}$-axis & $\mathrm{y}$-axis & z-axis \\
\hline centerline & $0.01 \%$ & $0.05 \%$ & $0.32 \%$ \\
\hline
\end{tabular}

Table 2: Maximum absolute differences of strain curves between SPH and glasgowHeart-IBFE for problem 1. A resolution of 4544 particles was used for the $\mathrm{SPH}$ simulation.

\section{Discussion}

The goal of this work was to evaluate the performance of the SPH method for cardiac mechanics. SPH simula-

\begin{tabular}{|l|l|l|l|}
\hline & Circ & Long & Trans \\
\hline Endo & $4.793 \%$ & $2.706 \%$ & $3.170 \%$ \\
\hline Epi & $1.259 \%$ & $2.879 \%$ & $2.169 \%$ \\
\hline Mid & $5.409 \%$ & $1.649 \%$ & $2.142 \%$ \\
\hline
\end{tabular}

Table 3: Maximum absolute differences of strain curves between SPH and glasgowHeart-IBFE for problem 2 . The nodes of the FE geometry were set as particles for the SPH simulation with a total of 18486 particles.

\begin{tabular}{|l|l|l|l|}
\hline & Circ & Long & Trans \\
\hline Endo & $5.038 \%$ & $8.701 \%$ & $4.755 \%$ \\
\hline Epi & $8.315 \%$ & $6.407 \%$ & $2.905 \%$ \\
\hline Mid & $16.168 \%$ & $4.672 \%$ & $3.814 \%$ \\
\hline
\end{tabular}

Table 4: Maximum absolute differences of strain curves between SPH and glasgowHeart-IBFE for problem 3 . The nodes of the FE geometry were set as particles for the SPH simulation with a total of 18486 particles.

tion results were compared against an available benchmark, where 11 FEM solvers were used to solve three mechanical problems. Comparison between SPH and FEM for these three experiments show that the proposed SPH simulation can be used for cardiac mechanics.

Indeed, it is observed that $\mathrm{SPH}$ approaches the FEM solutions for all problems. Both, the overall structure of the domains (beam and idealized ventricles in Fig. 1,5 and 9), as well as the centerlines (Fig. 2,6 and 10) are in good agreement, and deformation patterns are respected. Regarding strain, patterns and values of SPH and FEM are similar (see Fig. 4,8 and 12). Quantitatively, for Problem 1, Table 2 shows indeed similarities with strain measurements with highest differences up to $0.32 \%$ in the computation of strain in the $z$-axis direction. For problem 2, Table 3 shows that differences between the compared models are in most of the computed strains less than $5 \%$ and just in the circumferential midwall strain there is a maximum difference of up to $5.4 \%$. Finally, an analysis of the strain differences between SPH and FEM for problem 3 is shown in Table 4 . The highest differences between the two methods are less than $10 \%$ for the comparison of all strains except the circumferential midwall strain with a difference of up to $16.17 \%$. The three tables show that both methods are comparable and the worse results are the circumferential midwall strains, which probably arises from the fact that particles in SPH tend to get reorganized (without preserving their relative position) inside the volumetric object to keep the conservation of mass and the incompressibility constraints. In zones where particle reorganization gets relatively important, addi- 
Circ
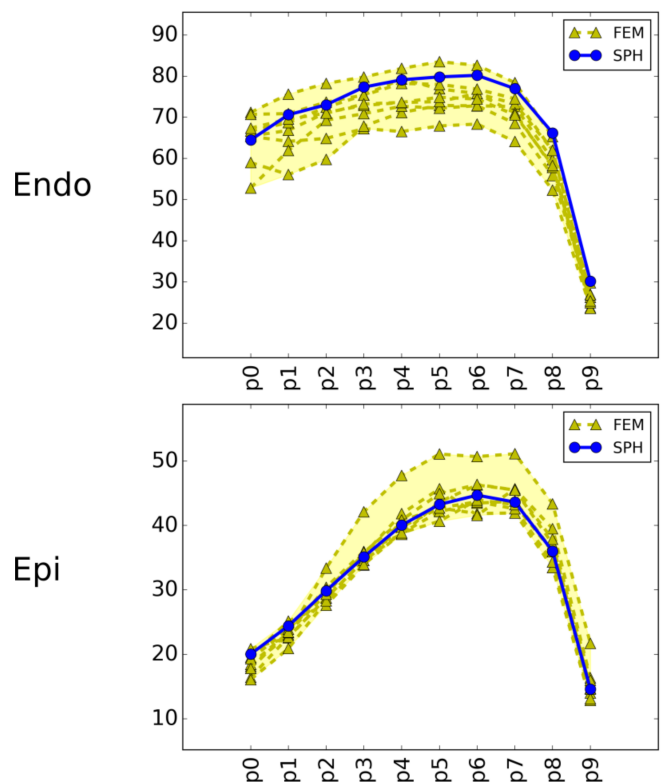

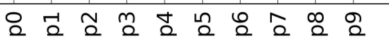

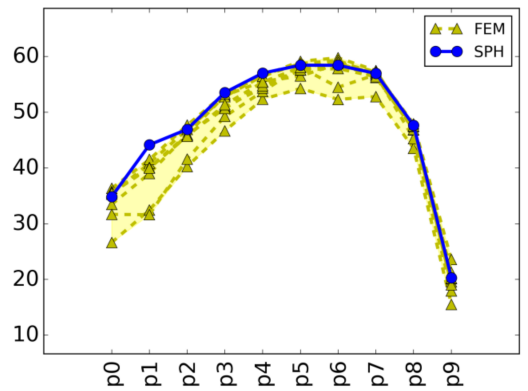

Long
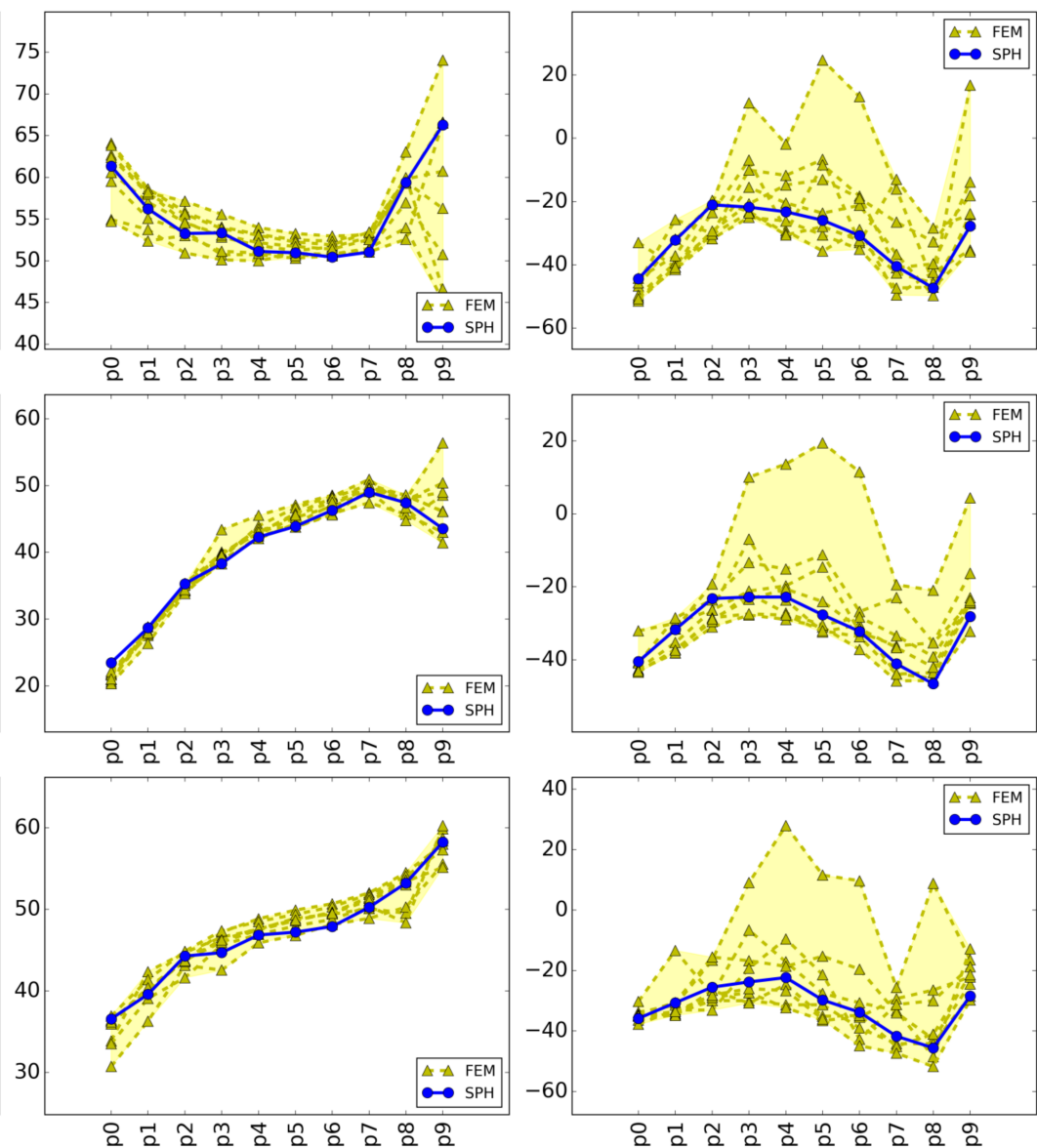

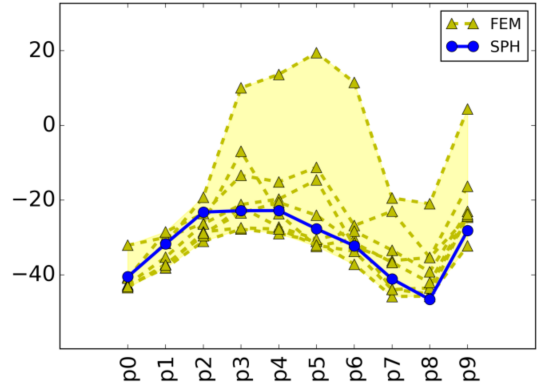

Trans

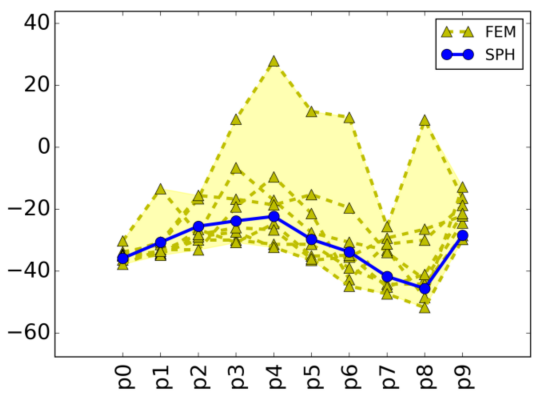

Fig. 8: Strain comparison for both FEM and SPH simulations of problem 2 in the three directions (circumferential, longitudinal and transversal) for each of the three myocardial layers taken into account (endocardial, epicardial and midwall). Solutions from all $11 \mathrm{FE}$ solvers published in [22] are overlaid with our SPH solution.

tional numerical strategies, such as particle reseeding or updating neighbors (also known as updated total Lagrangian) might be used [24]. The use of those strategies was not investigated here.

Even though differences in the results for meshbased and meshless methods are present, it is important to note that differences were higher among different mesh-based methods as it was reported in the benchmark [22]. For example, in problem 2 and 3, differences up to $20 \%$ in the endocardial circumferential strain were present.

These results were obtained thanks to the combination of several novelties that are available for SPH:

- A total Lagrangian formulation of the cardiac mechanics problem was formulated. We found that the method lends itself well to the implementation of exponential constitutive laws, commonly employed in cardiac tissue mechanics.
- The CSPM correction was implemented because it accounts for first order completeness and at the same time there is no need of ghost particles around the boundaries.

- The RK4 time integration scheme was used since it was reported to be a suitable time integration scheme in the context of SPH. [18]. Improvements of RK4 integration time exist but they are computationally more expensive [28, 18] and for the problems taken into account throughout this study, RK4 suffices.

- To deal with high deformations, the term of the artificial viscosity introduced by Monaghan [33] was added to the SPH formulation since the hourglass effect does not account for large deformations [14].

- Moreover, the ability to use a Poisson ratio of 0.495 to impose quasi-incompressibility showed robustness of the method. 

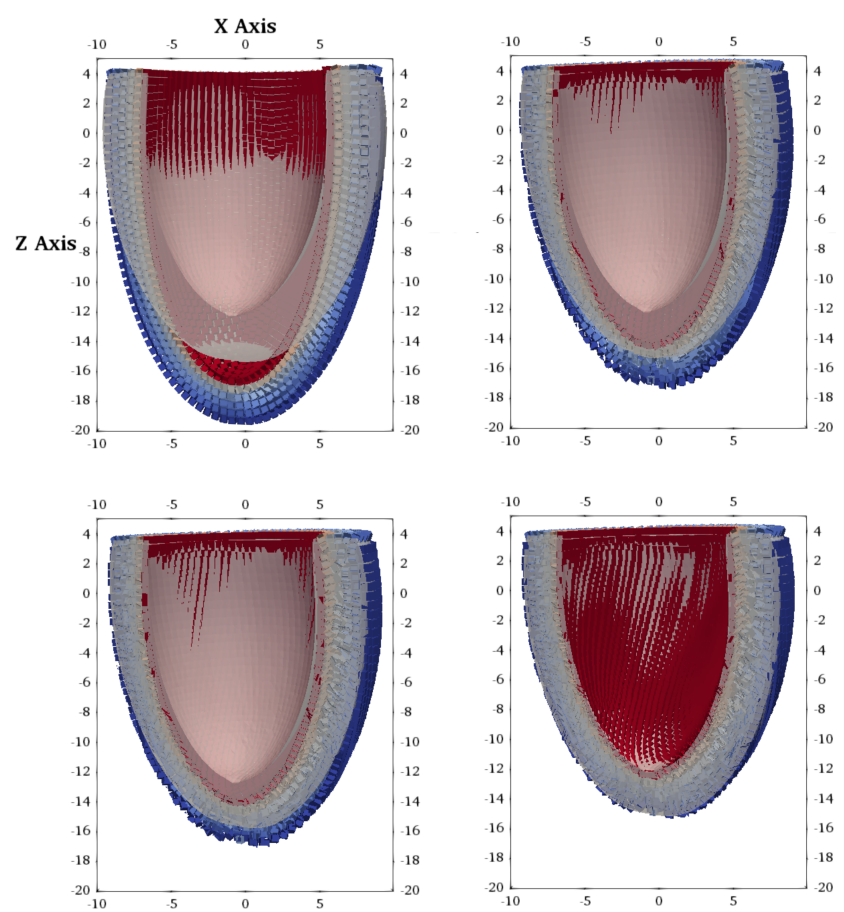

Fig. 9: Dynamic simulation of a ventricle subjected to dilation and contraction with the selected FEM solution as a reference (in grey). Endocardial pressure is increased gradually from phase A to phase D until $15 \mathrm{kPa}$ where the final state is reached. An active force on the fiber orientation is as well gradually increased from phase A to phase D until $60 k P a$.

$\mathrm{SPH}$ as a numerical solver for mechanics provides several advantages, starting from its meshless nature. In FE analysis, the polynomial bases are limited by the element structure, which implies that the method is less adaptive to different geometries. For complicated geometries like the one of the heart, building the element structure for high order elements (meshing) is very complicated and cannot be done automatically, so meshing and remeshing are very time consuming and labor-intensive.

By using SPH, the polynomial bases for spatial approximation are not limited by the element structure, avoiding the tedious step of meshing and remeshing [41]. On top of that, releasing the need for a mesh can certainly suit biophysical problems. In particular for cardiac mechanics, spatial resolution of images and image segmentations provide uncertainties on the wall location. Besides, small structures such as cardiac trabeculations can be hard to mesh.

The particle distribution was not evaluated in this article for all problems, since it was assumed that the same prior knowledge that is employed to mesh a geometry for FEM can be used for SPH. This FEM mesh

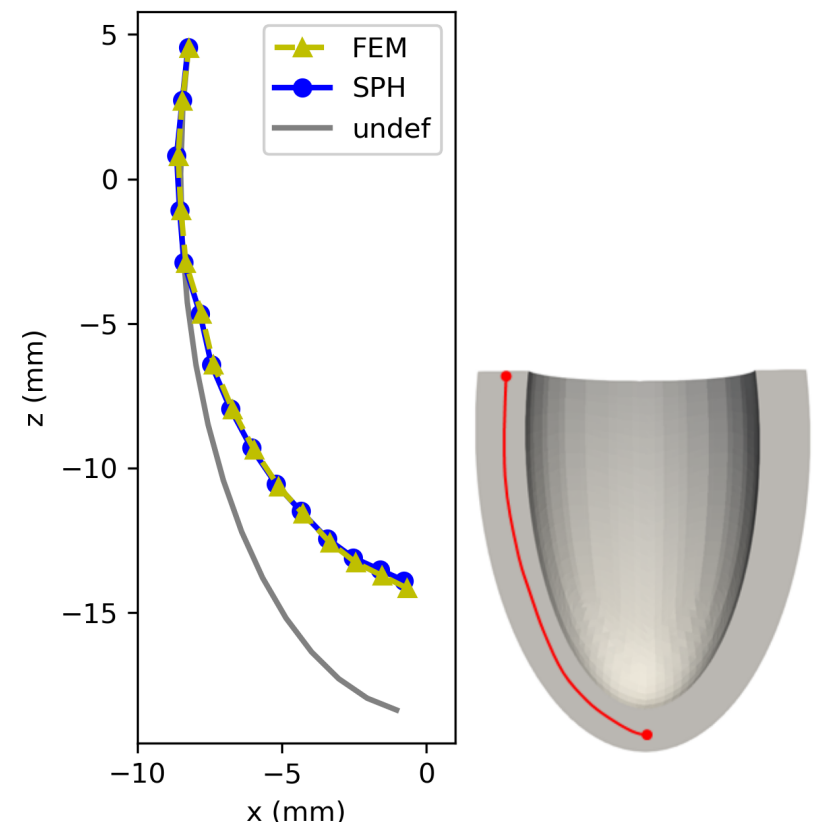

Fig. 10: Left: Comparison of the longitudinal centerline in the middle of the wall of problem 3 before (in grey) and after deformation for the SPH (in blue) and for the FEM (in dashed yellow line) simulations. Right: Undeformed mesh (in grey) with centerline (in red).

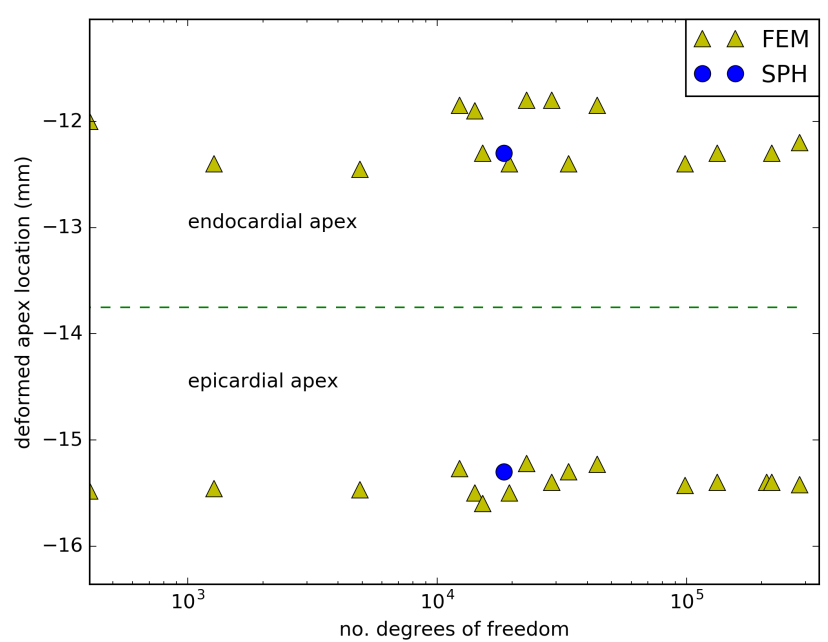

Fig. 11: Endocardial and Epicardial apex points for FEM and SPH simulations of problem 3 with different degrees of freedom.

might not be the optimal for SPH, but it can be used as a starting point towards an optimal strategy to find the particle number and distribution using SPH for cardiac mechanics.

Another advantage of SPH is the easy parallelization. The code could be further optimized in a more 
Circ
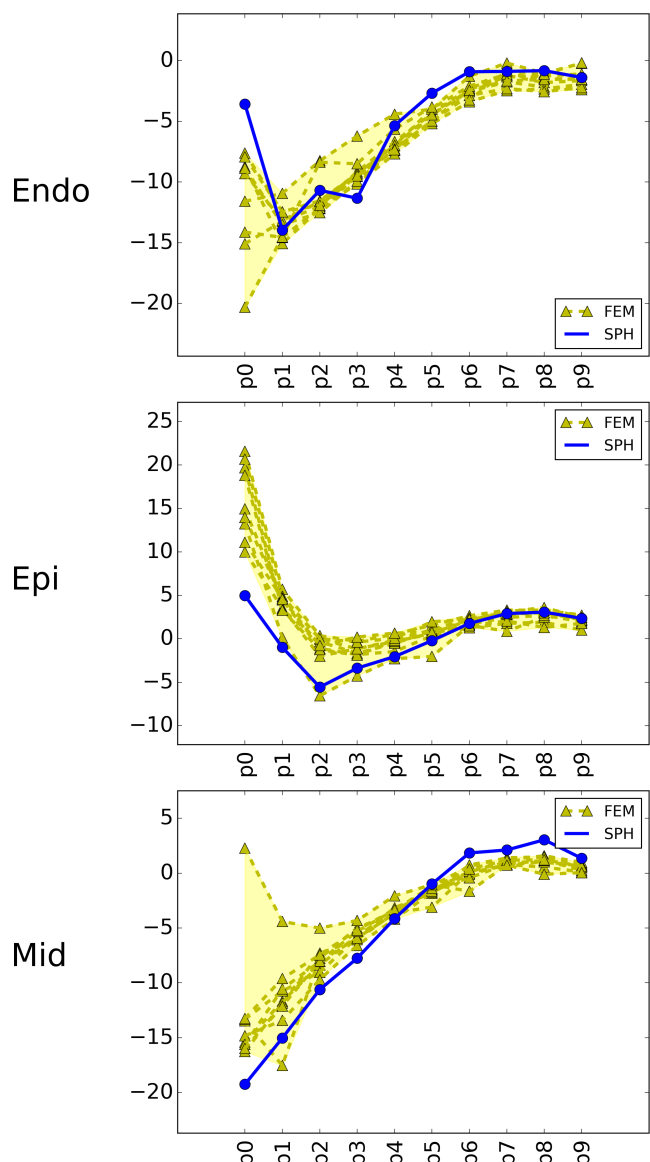

Long

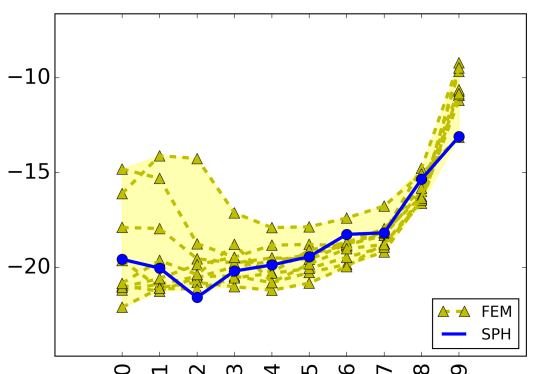

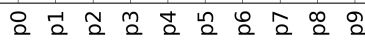
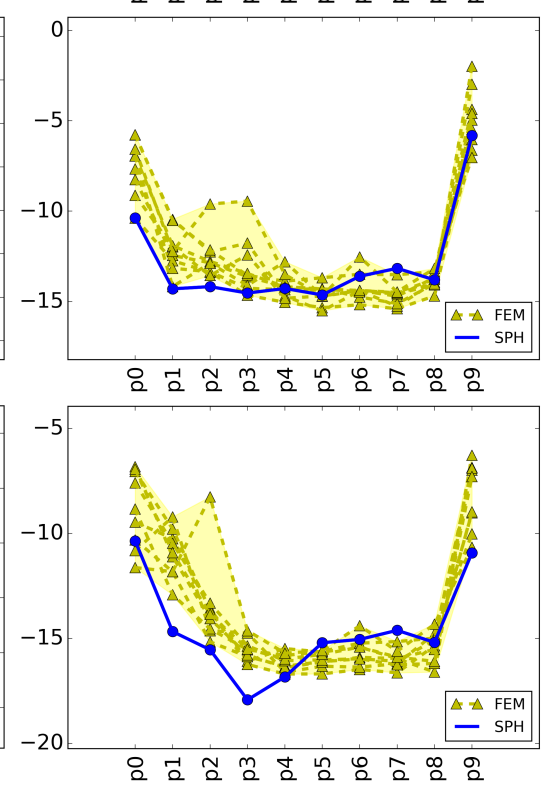

Trans

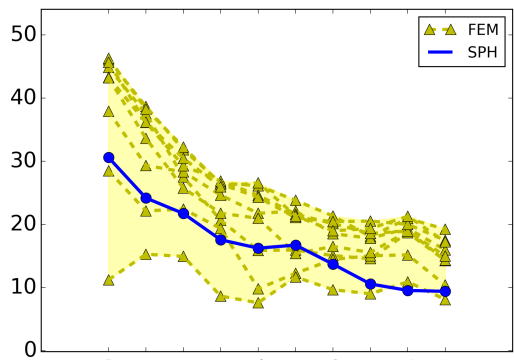

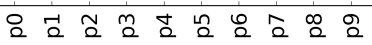

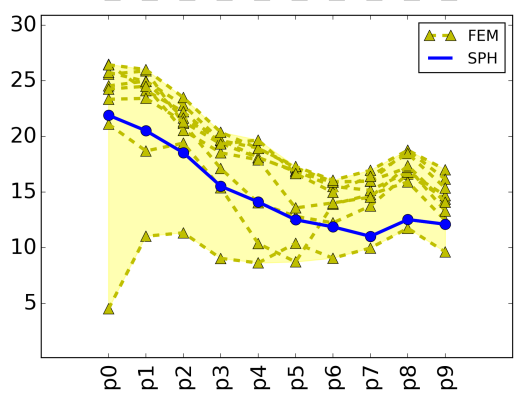

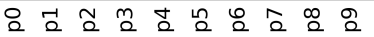

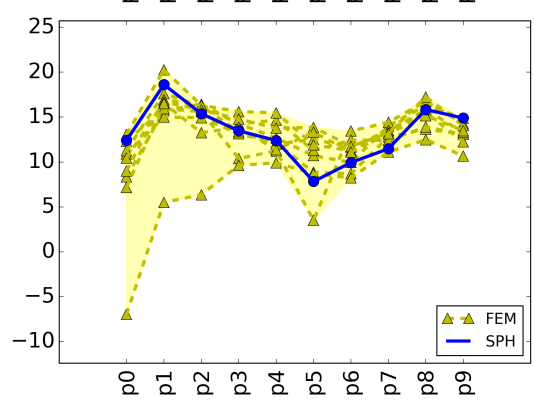

Fig. 12: Strain comparison for both FEM and SPH simulations of problem 3 in the three directions (circumferential, longitudinal and transversal) for each of the three myocardial layers taken into account (endocardial, epicardial and midwall). Solutions from all 11 FE solvers published in [22] are overlaid with our SPH solution.

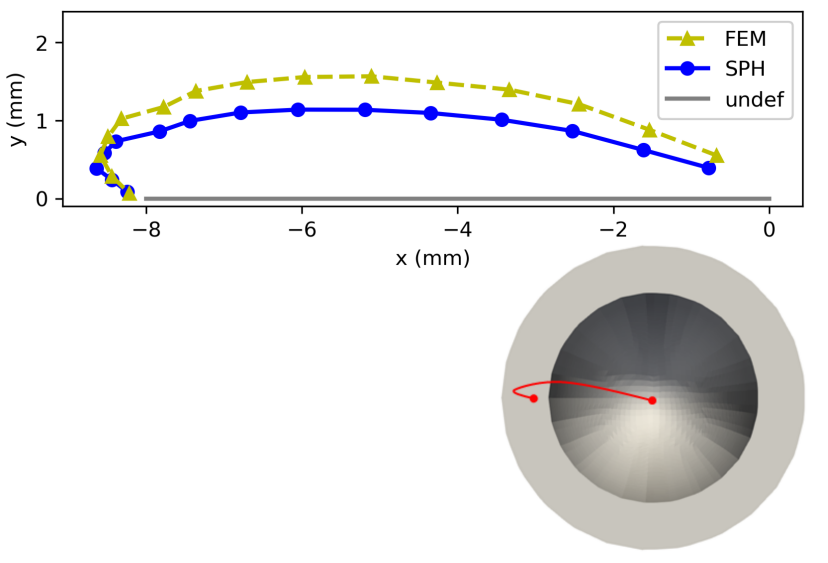

Fig. 13: Up: Circumferential centerlines of problem 3 to observe the difference on rotation between the compared SPH and FEM simulation. Bottom: View of the circumferential centerline from the base. efficient framework and parallelized using graphic processing units (GPU) that would probably decrease the computation time getting closer to real-time simulations (see [20] for an example with real time simulations with SPH). In fact, [19] discussed the potential of meshless total Lagrangian explicit methods such as SPH for real-time simulations.

A third advantage is the possibility to perform FSI for cardiac physiology. Indeed, SPH has been used for cardiac electrophysiology [27] and also used to simulate flow through valves [29]. Moreover, pure SPH for FSI has been shown to nicely perform when compared with experimental data [2]. The use of a unique numerical technique for cardiac electromechanics and fluid dynamics opens the possibility to tackle the challenging problem for cardiac modeling with a sole numerical technique.

As limitations of this work, without any GPU parallelization, the computation time using the same degrees 
of freedom as the ones using FEM is higher. Another limitation is that the problems taken into account did not reflect reality, especially in terms of deformation and complexity of the geometry. The solver needs to be evaluated on image-based heart anatomies. More research should be performed to properly characterize the magnitude of the errors for more complex cases, including an analysis of the influence of the choice of the time step. Finally, the main future direction is to couple the proposed model with an electrophysiological model, as well as with fluid particles.

\section{Conclusions}

In conclusion, the first cardiac mechanics solver based on $\mathrm{SPH}$ is presented in this paper. SPH initially suffered from a number of challenges that limited its use in solid mechanics. In this study, we have demonstrated that the SPH method can now be considered a more useful numerical tool in solid mechanics, and in particular in cardiac tissue mechanics, especially where complex geometries and large deformations are present.

\section{Acknowledgements}

The work is supported by the European Union Horizon 2020 research and innovation programme under grant agreement No 642676 (CardioFunXion).

\section{References}

1. Allan F. Bower: Applied Mechanics of Solids. CRC Press (2010)

2. Antoci, C., Gallati, M., Sibilla, S.: Numerical simulation of fluid-structure interaction by SPH. Computers and Structures 85, 879-890 (2007)

3. Babuška, I., Banerjee, U., Osborn, J.E., Zhang, Q.: Effect of numerical integration on meshless methods. Comput. Methods Appl. Mech. Eng. 198(3740), 2886-2897 (2009)

4. Belytschko, T., Krongauz, Y., Dolbow, J., Gerlach, C.: On the completeness of meshfree particle methods. Int. J. Numer. Methods Eng. 43(5), 785-819 (1998)

5. Biglino, G., Capelli, C., Bruse, J., Bosi, G.M., Taylor, A.M., Schievano, S.: Computational modelling for congenital heart disease: How far are we from clinical translation? Heart 103, 98-103 (2017)

6. Bonet, J., Kulasegaram, S., Rodriguez-Paz, M.X., Profit, M.: Variational formulation for the smooth particle hydrodynamics (SPH) simulation of fluid and solid problems. Comput. Methods Appl. Mech. Eng. 193(12-14), 1245-1256 (2004)

7. Bonet, J., Lok, T.S.: Variational and momentum preservation aspects of Smooth Particle Hydrodynamic formulations. Comput. Methods Appl. Mech. Eng. 180(1-2), 97-115 (1999)

8. Chabiniok, R., Wang, V.Y., Hadjicharalambous, M., Asner, L., Lee, J., Sermesant, M., Kuhl, E., Young, A.A., Moireau, P., Nash: Multiphysics and multiscale modelling, data-model fusion and integration of organ physiology in the clinic: ventricular cardiac mechanics. Interface Focus 6(2), 20150,083 (2016)

9. Chen, J.K., Beraun, J.E., Carney, T.C.: A corrective smoothed particle method for boundary value problems in heat conduction. Int. J. Numer. Methods Eng. 46(2), 231-252 (1999)

10. Davies, M.R., Wang, K., Mirams, G.R., Caruso, A., Noble, D., Walz, A., Lavé, T., Schuler, F., Singer, T., Polonchuk, L.: Recent developments in using mechanistic cardiac modelling for drug safety evaluation. Drug Discovery Today 21(6) (2016)

11. Douillet-Grellier, T., Pramanik, R., Pan, K., Albaiz, A., Jones, B.D., Williams, J.R.: Development of stress boundary conditions in smoothed particle hydrodynamics $(\mathrm{SPH})$ for the modeling of solids deformation. Comput. Part. Mech. pp. 1-21 (2016)

12. Duchateau, N., Sermesant, M., Delingette, H., Ayache, N.: Model-based generation of large databases of cardiac images: synthesis of pathological cine MR sequences from real healthy cases. IEEE Transactions on Medical Imaging (2017)

13. Dyka, C.T., Ingel, R.P.: An approach for tension instability in smoothed particle hydrodynamics (SPH). Comput. Struct. 57(4), 573-580 (1995)

14. Ganzenmueller, G.C.: An hourglass control algorithm for Lagrangian Smooth Particle Hydrodynamics. Comput. Methods Appl. Mech. Eng. 286, 87-106 (2015)

15. Gao, H., Wang, H., Berry, C., Luo, X., Griffith, B.E.: Quasi-static image-based immersed boundary-finite element model of left ventricle under diastolic loading. Int. j. numer. method. biomed. eng. 30(11), 1199-1222 (2014)

16. Guccione, J.M., Costa, K.D., McCulloch, a.D.: Finite element stress analysis of left ventricular mechanics in the beating dog heart. J. Biomech. 28(10), 1167-1177 (1995)

17. Han, L., Hu, X.: Sph modeling of fluid-structure interaction. Journal of Hydrodynamics 30, 62-69 (2018)

18. He, L., Seaid, M.: A Runge-Kutta-Chebyshev SPH algorithm for elastodynamics. Acta Mech. 227(7), 
1813-1835 (2016)

19. Horton, A., Wittek, A., Joldes, G.R., Miller, K.: A meshless Total Lagrangian explicit dynamics algorithm for surgical simulation. Int. j. numer. method. biomed. eng. 26, 977-998 (2010)

20. Krog, Ø.E., Elster, A.C.: Fast GPU-Based fluid simulations using SPH. In: Lecture Notes in Computer Science (including subseries Lecture Notes in Artificial Intelligence and Lecture Notes in Bioinformatics), vol. 7134 LNCS, pp. 98-109 (2012)

21. Land, S., et al.: Repository for the cardiac mechanics benchmark. http://www.bitbucket.org/ sander314/mechbench. Accessed: 2018-01-20

22. Land, S., et al.: Verification of cardiac mechanics software: benchmark problems and solutions for testing active and passive material behaviour. Proc. Math. Phys. Eng. Sci. 471(2184), 20150,641 (2015)

23. Lang, R.M., Badano, L.P., Mor-Avi, V., Afilalo, J., Armstrong, A., Ernande, L., Flachskampf, F.A., Foster, E., Goldstein, S.A., Kuznetsova, T., Lancellotti, P., Muraru, D., Picard, M.H., Rietzschel, E.R., Rudski, L., Spencer, K.T., Tsang, W., Voigt, J.U.: Recommendations for cardiac chamber quantification by echocardiography in adults: An update from the American society of echocardiography and the European association of cardiovascular imaging. European Heart Journal Cardiovascular Imaging 16, 233-271 (2015)

24. Leroch, S., Varga, M., Eder, S.J., Vernes, A., Rodriguez Ripoll, M., Ganzenmüller, G.: Smooth particle hydrodynamics simulation of damage induced by a spherical indenter scratching a viscoplastic material. Int. J. Solids Struct. 81(December), 188-202 (2016)

25. Libersky, L.D., Petschek, A.G.: Smooth particle hydrodynamics with strength of materials. In: Adv. Free. Method Incl. Contrib. Adapt. Gridding Smooth Part. Hydrodyn. Method, pp. 248257. Springer Verlag Berlin Heidelberg (1991)

26. Lin, J., Naceur, H., Coutellier, D., Laksimi, A.: Efficient meshless SPH method for the numerical modeling of thick shell structures undergoing large deformations. Int. J. Non. Linear. Mech. 65, 1-13 (2014)

27. Lluch, È., Doste, R., Giffard-Roisin, S., This, A., Sermesant, M., Camara, O., De Craene, M., Morales, H.G.: Smoothed particle hydrodynamics for electrophysiological modeling: An alternative to finite element methods. In: Lecture Notes in Computer Science (including subseries Lecture Notes in Artificial Intelligence and Lecture Notes in Bioinformatics), vol. 10263 LNCS, pp. 333-343 (2017)
28. Mabssout, M., Herreros, M.I.: Runge-Kutta vs Taylor-SPH: Two time integration schemes for SPH with application to Soil Dynamics. Appl. Math. Model. 37(5), 3541-3563 (2013)

29. Mao, W., Caballero, A., McKay, R., Primiano, C., Sun, W.: Fully-coupled fluid-structure interaction simulation of the aortic and mitral valves in a realistic 3D left ventricle model. PLoS ONE 12(9) (2017)

30. Mao, W., Li, K., Sun, W.: Fluid-Structure Interaction Study of Transcatheter Aortic Valve Dynamics Using Smoothed Particle Hydrodynamics. Cardiovascular Engineering and Technology 7(4), 374-388 (2016)

31. Marchesseau, S., Sermesant, M., Billet, F., Delingette, H., Ayache, N.: Personalization of electromechanical models of the cardiac ventricular function by heterogeneous clinical data assimilation. Multi-modality Card. Imaging Process. Anal. (2015)

32. Monaghan, J.J.: SPH without a Tensile Instability. J. Comput. Phys. 159(2), 290-311 (2000)

33. Monaghan, J.J.: Smoothed particle hydrodynamics. Reports Prog. Phys. 68(8), 1703 (2005)

34. Naceur, H., Lin, J., Coutellier, D., Laksimi, A.: Efficient smoothed particle hydrodynamics method for the analysis of planar structures undergoing geometric nonlinearities. J. Mech. Sci. Technol. 29(5), 2147-2155 (2015)

35. Ogden, R.: Nonlinear Mechanics of Soft solids Including Biological Tissues. The 5th Winter School on Appl. Math. Univ. Hong Kong (2011)

36. Rabczuk, T., Belytschko, T., Xiao, S.P.: Stable particle methods based on Lagrangian kernels. Comput. Methods Appl. Mech. Eng. 193(12-14), 10351063 (2004)

37. Rausch, M.K., Karniadakis, G.E., Humphrey, J.D.: Modeling Soft Tissue Damage and Failure Using a Combined Particle/Continuum Approach. Biomech. Model. Mechanobiol. 16(1), 249-261 (2017)

38. Sugiura, S., Washio, T., Hatano, A., Okada, J., Watanabe, H., Hisada, T.: Multi-scale simulations of cardiac electrophysiology and mechanics using the University of Tokyo heart simulator. Progress in Biophysics and Molecular Biology 110, 380-389 (2012)

39. Swegle, J.W., Hicks, D.L., Attaway, S.W.: Smoothed Particle Hydrodynamics Stability Analysis. J. Comput. Phys. 116(1), 123-134 (1995)

40. Wong, K.C., Wang, L., Zhang, H., Liu, H., Shi, P.: Meshfree implementation of individualized active 
cardiac dynamics. Computerized Medical Imaging and Graphics 34(1), 91-103 (2010)

41. Zhang, L.W., Ademiloye, A.S., Liew, K.M.: Meshfree and Particle Methods in Biomechanics: Prospects and Challenges. Arch. Comput. Methods Eng. 2 (2018) 